CARDIOVASCULAR MEDICINE

\title{
Cholinergic stimulation with pyridostigmine protects against exercise induced myocardial ischaemia
}

\author{
R R T Castro, G Porphirio, S M Serra, A C L Nóbrega
}

Heart 2004;90:1119-1123. doi: 10.1136/hrt.2003.028167

See end of article for authors' affiliations

\section{Correspondence to:}

Dr A C L Nóbrega, Rua Cinco de Julho, 318 /

1001, Niterói, RJ 24220-

111,Brazil; anobrega@

urbi.com.br

Accepted

26 February 2004
Objective: To determine the acute effects of pyridostigmine bromide, a reversible cholinesterase inhibitor, during exercise in patients with coronary artery disease.

Design: Double blind, randomised, placebo controlled, crossover study.

Setting: Outpatients evaluated in an exercise test laboratory.

Patients: 15 patients with exercise induced myocardial ischaemia.

Interventions: Maximal cardiopulmonary exercise test on a treadmill according to an individualised ramp protocol on three days. The first day was used for adaptation to the equipment and to determine exercise tolerance and the presence of exercise induced ischaemia. On the other two days, the cardiopulmonary exercise test was performed two hours after oral administration of pyridostigmine $(45 \mathrm{mg})$ or placebo. All patients were taking their usual medication during the experiments.

Main outcome measures: Rate-pressure product and oxygen uptake during exercise.

Results: Pyridostigmine inhibited the submaximum chronotropic response ( $p=0.001)$, delaying the onset of myocardial ischaemia, which occurred at a similar rate-pressure product (mean (SE) placebo 20.55 (1.08) $\mathrm{mm} \mathrm{Hg} \times$ beats $/ \mathrm{min} 10^{3}$; pyridostigmine 19.75 (1.28) $\mathrm{mm} \mathrm{Hg} \times$ beats $/ \mathrm{min} 10^{3} ; \mathrm{p}=0.27$ ) but at a higher exercise intensity (oxygen consumption: placebo $18.6(1.7) \mathrm{ml} / \mathrm{kg} / \mathrm{min}$; pyridostigmine 19.6 (1.8) $\mathrm{ml} / \mathrm{kg} / \mathrm{min} ; \mathrm{p}=0.03$ ). Also, pyridostigmine increased peak oxygen consumption (placebo 23.6 (2) $\mathrm{ml} / \mathrm{kg} / \mathrm{min}$; pyridostigmine $24.8(2) \mathrm{ml} / \mathrm{kg} / \mathrm{min} ; \mathrm{p}=0.01$ ) and peak oxygen pulse (placebo 12.9 (1) $\mathrm{ml} /$ beat; pyridostigmine 13.6 (1) $\mathrm{ml} /$ beat; $p=0.02$ ).

Conclusions: Pyridostigmine improved peak exercise tolerance and inhibited the chronotropic response to submaximum exercise, increasing the intensity at which myocardial ischaemia occurred. These results suggest that pyridostigmine can protect against exercise induced myocardial ischaemia.
$\mathrm{D}$ ysfunction of the autonomic nervous system increases the risk of arrhythmic events and sudden death after acute myocardial infarction. ${ }^{2}$ Treatment options to improve sympathovagal balance have concentrated mainly on reducing sympathetic effects. The concept that adrenergic hyperactivity can be deleterious has led to the widespread clinical use of $\beta$ blockers in patients after myocardial infarction, with a consequent reduction in overall mortality. ${ }^{3}$ Decreased parasympathetic activity also is an independent risk factor after an acute myocardial infarction ${ }^{5}$ but few studies have investigated treatment alternatives for parasympathetic dysfunction. ${ }^{6}$

Pyridostigmine bromide is a reversible anticholinesterase agent that exerts an indirect cholinergic stimulation. In healthy volunteers, pyridostigmine decreased resting heart rate, ${ }^{7}$ increased heart rate variability, ${ }^{8}$ and blunted the cardiac response to mental stress ${ }^{9}$ and to dynamic exercise. ${ }^{10}$ In the clinical setting, pyridostigmine has been shown to increase heart rate variability and to decrease the density of ventricular arrhythmia ${ }^{11}$ in patients with heart failure. However, the effects of pyridostigmine in patients with coronary artery disease are largely unknown.

Exercise testing is a useful tool to evaluate patients with coronary artery disease both to investigate the occurrence of exercise induced ischaemia and to determine peak oxygen consumption. ${ }^{12}$ The intensity of exercise beyond which a patient develops myocardial ischaemia should guide recommendations regarding daily life activities. In addition, the peak oxygen consumption during exercise is an objective measure of maximum aerobic power, which has been shown to have a strong inverse association with cardiovascular death. ${ }^{13}$ Since myocardial ischaemia results from an imbalance between coronary blood flow and cardiac oxygen demand, both being modulated by autonomic tone, drugs that mimic autonomic action may cause multiple effects on the haemodynamic responses to exercise, including the occurrence of myocardial ischaemia. We hypothesised that by reducing the chronotropic response to progressive exercise, pyridostigmine would delay the increase in myocardial oxygen demand and thus the onset of ischaemia. Therefore, the purpose of this study was to determine the acute effects of pyridostigmine on the integrated physiological responses and the occurrence of myocardial ischaemia during dynamic exercise in patients with coronary artery disease and exercise induced myocardial ischaemia.

\section{METHODS \\ Patients}

Nineteen patients with a history of angina pectoris were selected for participation in the study. Only 15 of them had exercise induced angina, ST depression, or both, determined in the evaluation test preceding the experimental days, and were eligible for the study (12 men; mean (SD) age 65 (8) years; body mass index $27(3) \mathrm{kg} / \mathrm{m}^{2}$ ). Eight of them had a previous acute myocardial infarction. All patients gave written informed consent to participate in the study after full explanation of the procedures and their potential risks. None of the patients had diabetes mellitus or other metabolic diseases, renal failure, left ventricular fraction $<40 \%$, a pacemaker, or autonomic dysfunction.

Abbreviations: $\dot{\mathrm{V}} \mathrm{CO}_{2}$, carbon dioxide production; $\dot{\mathrm{V}}_{\mathrm{E}}$, minute ventilation; $\dot{\mathrm{V}}_{2}$, oxygen consumption 
The investigation conforms with the principles outlined in the Declaration of Helsinki and was approved by the institutional research ethics committee on human research. All participants continued taking their usual medication throughout the study period. They were instructed to avoid alcohol, beverages containing caffeine, and strenuous physical activity the day before the experiments.

\section{Protocol}

Each patient underwent, on three different days, a maximal exercise test on a treadmill (KT10200; Inbramed, Porto Alegre, Brazil) according to an individualised ramp protocol, where the initial and final work rates were set to achieve an estimated test duration of 8-12 minutes, considering the individual clinical condition and physical activity habits of each patient. The exercise test was interrupted if the volunteer developed angina (grade 3, out of a maximum of 4), exhaustion, complex arrhythmia, ST segment elevation $(\geqslant 2 \mathrm{mV})$, ST segment depression $>3 \mathrm{mV}$, or a fall in blood pressure. Expiratory gases and pulmonary ventilation were automatically determined (Teem 100, Aerosport, Ann Arbor, Michigan, USA; see details below). The first day was used for adaptation to the equipment and to determine exercise tolerance and the presence of exercise induced ischaemia. Each study day was separated from the next one by at least a 48 hour period for washout of the drug used. During the other two days, the exercise test was performed according to the same protocol, two hours after the oral administration of either pyridostigmine $(45 \mathrm{mg})$ or placebo, following a randomised, cross over, double blind protocol. We controlled the potential effect of drug sequence by using a latin square design, where patients were randomly, but evenly $(50 \%-$ $50 \%$ ), assigned to placebo-pyridostigmine or pyridostigmineplacebo.

The 12 lead ECG was continuously monitored throughout the exercise test (with Mason-Likar electrode placement). ECG tracings and arterial blood pressure (measured with a cuff sphygmomanometer) were recorded before the exercise test, every minute during exercise, and during the recovery phase. Myocardial ischaemia was defined as ST depression of $\geqslant 1 \mathrm{mV}$ measured at 0.08 seconds after the $\mathrm{J}$ point.

Oxygen consumption $\left(\dot{\mathrm{V}}_{2}\right)$, carbon dioxide production $\left(\dot{\mathrm{V}} \mathrm{CO}_{2}\right)$, and minute ventilation $(\dot{\mathrm{V}} \mathrm{E})$ were registered every 20 seconds. Derived variables were calculated on line (Aerograph, USA). Anaerobic threshold was identified by two experienced evaluators by the combination of the following methods ${ }^{14}$ : firstly, at the point of upward inflection of the ventilation $\mathrm{X}$ time curve; secondly, at the beginning of a consistent increase in the ventilatory equivalent for $\mathrm{O}_{2}\left(\dot{\mathrm{V}}_{\mathrm{E}} /\right.$ $\dot{\mathrm{V}}_{2}$ ) without a concomitant increase in the ventilatory equivalent for carbon dioxide $\left(\dot{\mathrm{V}} \mathrm{E} / \dot{\mathrm{V}}_{2}\right)$; and thirdly, at the beginning of an increase in expired oxygen fraction. Ventilatory threshold was considered to be the point identified by at least two of these three criteria. There was no case where each one of the criteria identified different thresholds.

\section{Statistical analysis}

The occurrence of adverse reactions was compared by the McNemar test. Heart rate and oxygen pulse values throughout various moments of exercise under the two conditions (after placebo and after pyridostigmine) were analysed by a two factor analysis of variance with repeated measures, where time (moment of exercise test) and drug (pyridostigmine, placebo) were the main factors. If a significant $F$ value was obtained, analysis of variance was followed by the Student-Newman-Keuls test for pairwise post hoc comparisons. In addition, we used paired $t$ tests to compare the variables measured at maximum effort with placebo and pyridostigmine. Significance was set at $\mathrm{p}<0.05$. Results are presented as mean (SE).

\section{RESULTS}

Table 1 shows patients' characteristics. There was no difference in side effects between placebo and pyridostigmine $(p=0.23)$. One patient reported transient abdominal discomfort and two others reported light headache after pyridostigmine ingestion. After placebo, one patient reported light headache and presented with atrial tachycardia and two others had a decrease in arterial blood pressure during exercise.

The exercise test was interrupted for similar reasons with placebo and pyridostigmine: exhaustion $(67 \%, \mathrm{n}=10)$ and angina $(33 \%, \mathrm{n}=05)$. During placebo use, two tests were interrupted because of hypotension and another because of supraventricular tachycardia.

Pyridostigmine increased exercise tolerance, corresponding to higher peak pulmonary ventilation, peak $\dot{\mathrm{V}}_{2}$, and peak oxygen pulse (table 2). Concerning the chronotropic response, pyridostigmine did not change peak heart rate $(\mathrm{p}=0.31)$ but inhibited the submaximum response to exercise $(p=0.001$; fig 1$)$. This slower chronotropic response delayed the onset of myocardial ischaemia, which occurred at a similar rate-pressure product $(\mathrm{p}=0.27)$ but at higher exercise intensity ( $p=0.03$; fig 2 ).

There was no difference in the peak arterial blood pressure after ingestion of placebo and pyridostigmine (table 2). The ventilatory threshold relative to peak $\dot{\mathrm{V}}_{2}$ was similar with placebo $(64.6 \%)$ and pyridostigmine $(62.4 \% ; \mathrm{p}=0.407)$.

The magnitude of ST depression was different $(p=0.02)$ after placebo $(2.38 \mathrm{mV})$ and pyridostigmine $(1.96 \mathrm{mV})$. When analysed separately, in patients with and those without symptomatic myocardial ischaemia there were no differences in the magnitude of ST depression after placebo and after pyridostigmine. Also, patients were analysed according to whether they were or were not taking $\beta$ blockers, and no difference was found in the magnitude of ST depression after placebo and after pyridostigmine. Compared with patients who were not taking $\beta$ blockers, patients who were taking $\beta$ blockers had more a pronounced increase of peak $\dot{\mathrm{V}}_{2}(\mathrm{p}=0.02)$ and $\dot{\mathrm{V}}_{\mathrm{O}_{2}}$ at the moment of myocardial ischaemia $(p=0.049)$ after pyridostigmine. There was no difference in double product at the moment of ischaemia after placebo and after pyridostigmine when these patients were analysed.

\section{Study limitations}

As table 1 shows, although all patients had exercise induced myocardial ischaemia, most of them were not taking a $\beta$ blocker or angiotensin converting enzyme inhibitor. For ethical reasons we could not change their prescription. Following a cross over protocol, each patient was his or her own control, which minimises the differences between patients' prescriptions. Also, a major concern about this study was that the variables may have improved after administration of pyridostigmine because most patients were not taking maximum tolerated doses. To test this hypothesis we decided to analyse separately patients who were and were not taking $\beta$ blockers. Surprisingly we found better results when pyridostigmine was used in combination with $\beta$ blocker.

Patients either with or without previous myocardial infarction were included but they were otherwise quite similar. More important, the responses to exercise were equivalent in both groups. For example, peak oxygen uptake, a major outcome of the study, was comparable between patients with and those without previous myocardial 
Table 1 Individual patient characteristics

\begin{tabular}{|c|c|c|c|c|c|}
\hline Patient & Sex & $\begin{array}{l}\text { Age } \\
\text { (years) }\end{array}$ & $\begin{array}{l}\text { BMI } \\
\left(\mathrm{kg} / \mathrm{m}^{2}\right)\end{array}$ & $\begin{array}{l}\text { Previous } \\
\text { AMI }\end{array}$ & Drugs in use \\
\hline 1 & $M$ & 69 & 27.8 & No & Calcium channel antagonist, aspirin \\
\hline 2 & M & 50 & 27.3 & Yes & Calcium channel antagonist, aspirin \\
\hline 3 & M & 63 & 21.9 & Yes & $\beta$ Blocker, nitrates, $\mathrm{ACE}$ inhibitor, aspirin \\
\hline 4 & $M$ & 63 & 25.4 & Yes & $\beta$ Blocker, nitrate, $A C E$ inhibitor, aspirin \\
\hline 5 & $M$ & 73 & 25.3 & No & Nitrate, flunarizine \\
\hline 6 & $M$ & 74 & 26.4 & No & Nitrate, ACE inhibitor, calcium channel antagonist \\
\hline 7 & $\mathrm{~F}$ & 67 & 29.1 & No & Nitrate, $\beta$ blocker \\
\hline 8 & $M$ & 48 & 22.8 & No & $\beta$ Blocker \\
\hline 9 & $M$ & 62 & 24.3 & Yes & Calcium channel antagonist, nitrate \\
\hline 10 & $M$ & 69 & 24.5 & No & Aspirin, lipid lowering agent \\
\hline 11 & $M$ & 57 & 23.3 & Yes & Nitrate, ACE inhibitor, $\beta$ blocker \\
\hline 12 & $\mathrm{~F}$ & 67 & 27.9 & Yes & Nitrate, ACE inhibitor, aspirin \\
\hline 13 & $M$ & 71 & 26.5 & No & Nitrate, ACE inhibitor, lipid lowering agent, diuretic \\
\hline 14 & M & 68 & 34.0 & Yes & Nitrate, ACE inhibitor \\
\hline 15 & $\mathrm{~F}$ & 66 & 30.4 & Yes & Nitrate, ACE inhibitor, lipid lowering agent, $\beta$ blocker \\
\hline
\end{tabular}

infarction both with placebo $(\mathrm{p}=0.728)$ and with pyridostigmine $(p=0.567)$.

\section{DISCUSSION}

The mechanism of action of pyridostigmine is characterised by the increased concentration of acetylcholine in the synaptic clefts through reversible inhibition of cholinesterase activity, thus enhancing parasympathetic tone. Although pyridostigmine has been used extensively by patients with myasthenia gravis to counteract skeletal muscle weakness, its cardiovascular action has been considered a side effect. ${ }^{6}$

The systematic study of its haemodynamic effects has shown that pyridostigmine decreases resting ${ }^{7}{ }^{15}$ and exercise heart rate ${ }^{10}$ and reduces QTc dispersion ${ }^{15}$ in healthy people without impairing exercise tolerance. ${ }^{10}$ When administered at eight hour intervals, pyridostigmine elicited sustained 24 hour bradycardia and augmented heart rate variability. ${ }^{8}$ All of these effects were obtained without impairment of systolic or diastolic cardiac functions. ${ }^{16}$ In addition, a single $45 \mathrm{mg}$ dose of pyridostigmine blunted the double product increase during mental stress when given orally to healthy people. ${ }^{9}$ Moreover, pyridostigmine inhibited the haemodynamic response to central adrenergic stimulation produced by intracerebroventricular injection of glutamate in a rat model. ${ }^{17}$ When patients with coronary artery disease were studied, pyridostigmine was shown to decrease the QTc interval at rest, ${ }^{18}$ an effect that may have protective implications, since increased QTc is associated with higher mortality after myocardial infarction. ${ }^{19}$ The present study expands these previous findings by analysing the effects of pyridostigmine on the cardiopulmonary response to exercise in patients with stable angina.

The effects of pyridostigmine on the haemodynamic responses to dynamic exercise have scarcely been studied. Arad et $a^{20}$ have shown that pyridostigmine $(30 \mathrm{mg}$, three times a day for two days) did not change the heart rate response to cycling in eight hypertensive volunteers. This is in contrast to the present study, where pyridostigmine caused submaximal bradycardia during running in healthy participants. Several factors could have accounted for this difference, such as the characteristics of the patients, type of ergometer used, protocol of administration of pyridostigmine, and smaller sample size in the study of Arad et al, ${ }^{20}$ which may have decreased the statistical power of the test used for comparison. On the other hand, in a previous study involving five healthy volunteers ${ }^{21}$ a single dose $(30 \mathrm{mg})$ of pyridostigmine inhibited the heart rate response to cycling, in accordance with our present results.

The present study is apparently at odds with another one, ${ }^{22}$ which found that the rate-pressure product at myocardial ischaemia was increased by intravenous atropine, a cholinergic blocker. Those authors suggested that this result may indicate an increase in myocardial blood flow similar to the effect of isosorbide dinitrate. However, the patients achieved the same exercise tolerance with atropine and placebo, contrary to the experiments with isosorbide that increased the time to ischaemia, which is the key aim when treating this group of patients.

It is important to compare the effects of pyridostigmine with those of other pharmacological strategies to provoke cholinergic activation. Previous studies with low dose atropine and scopolamine had promising results ${ }^{23}{ }^{24}$ but long term use would be limited by side effects through their action on the central nervous system. Pirenzepine is an $M_{1}$ selective antagonist devoid of central action, which can paradoxically augment parasympathetic tone when administered at low doses. The mechanism of this paradoxical effect is not completely understood and may be caused by enhancement of acetylcholine release after blockade of presynaptic

Table 2 Peak exercise data during placebo and pyridostigmine administration $(n=15)$

\begin{tabular}{|c|c|c|c|}
\hline Variable & Placebo & Pyridostigmine & p Value \\
\hline Time to exhaustion $(\min , \mathrm{s}))$ & $9^{\prime} 18^{\prime \prime}\left(18^{\prime \prime}\right)$ & $10^{\prime} 06^{\prime \prime}\left(18^{\prime \prime}\right)$ & 0.002 \\
\hline$\dot{\mathrm{V}} \mathrm{O}_{2}$ peak $(\mathrm{ml} / \mathrm{kg} / \mathrm{min})$ & $23.6(2.0)$ & $24.8(2.0)$ & 0.011 \\
\hline$\dot{\mathrm{V}} \mathrm{CO}_{2}(\mathrm{ml} / \mathrm{kg} / \mathrm{min})$ & $20.6(2.2)$ & $22.1(2.2)$ & 0.031 \\
\hline$\dot{\mathrm{V}}_{\mathrm{E}}(\mathrm{l} / \mathrm{min})$ & $57.4(6.0)$ & $61.9(6.3)$ & 0.024 \\
\hline Oxygen pulse (ml/beat) & $12.9(1)$ & $13.6(1)$ & 0.017 \\
\hline Heart rate (beats/min) & $129(5)$ & $129(5)$ & 0.313 \\
\hline Systolic BP (mm Hg) & $192(9)$ & $194(9)$ & 0.349 \\
\hline Diastolic BP (mm Hg) & $97(4)$ & $92(5)$ & 0.159 \\
\hline
\end{tabular}




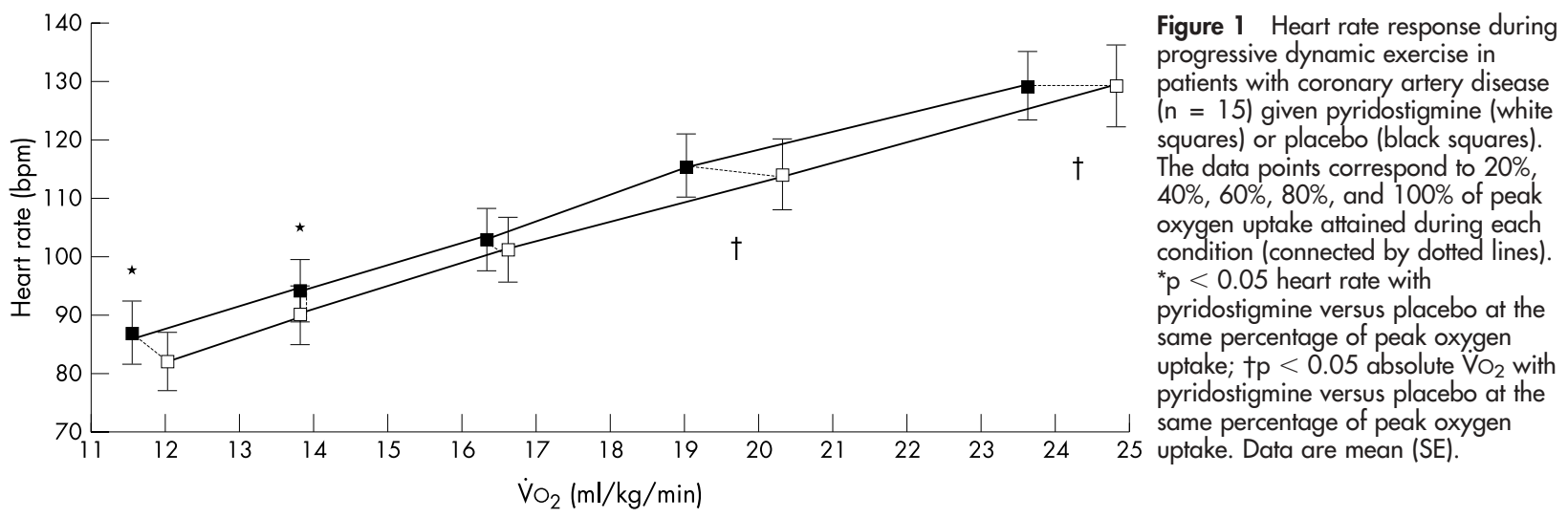

muscarinic autoreceptors. ${ }^{25}$ Although the paradoxical effect of pirenzepine has been documented for doses ranging between 0.01 and $0.1 \mathrm{mg} / \mathrm{kg}$, other studies have shown that higher doses may augment parasympathetic tone. ${ }^{26} 27$ Marraccini et $\mathrm{l}^{28}$ have shown that a single intravenous dose of pirenzepine $(2 \mathrm{mg}$ ) caused relative bradycardia during mild levels of exercise, improving exercise tolerance in patients with effort ischaemia. It is clear from the results of this study ${ }^{28}$ that pirenzepine may induce its paradoxical effect even when higher doses $(>0.1 \mathrm{mg} / \mathrm{kg})$ of the drug are administered. Therefore, the study by Marraccini et al..$^{28}$ supports our present results, showing that drugs that can enhance parasympathetic tone can blunt exercise induced myocardial ischaemia in patients with myocardial ischaemia. A recently published study ${ }^{29}$ has tested the effects of pirenzepine $(0.1 \mathrm{mg} / \mathrm{kg})$ in dogs after myocardial infarction. This dose was shown to increase heart rate variability but the
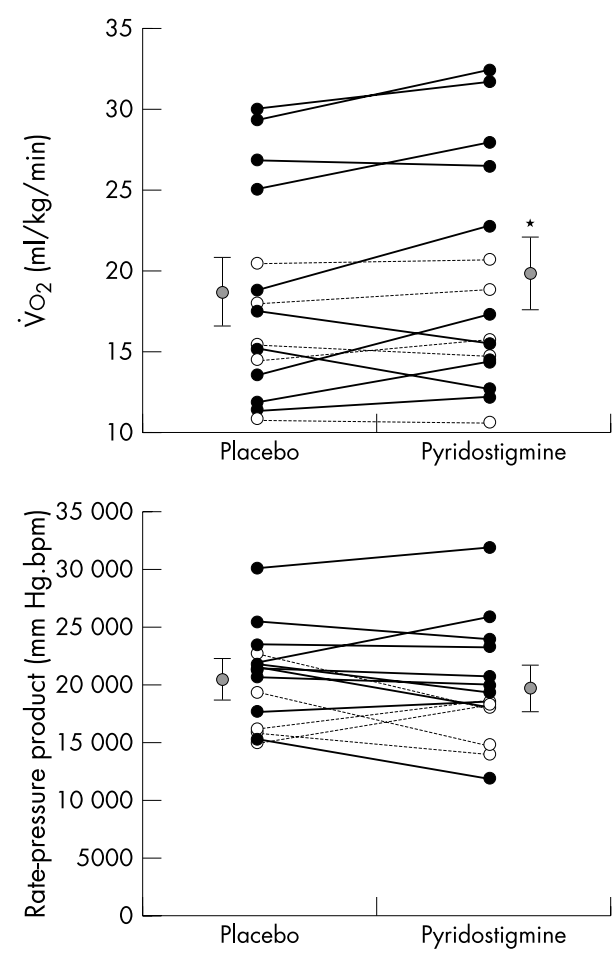

Figure 2 (Top) Oxygen uptake and (bottom) rate-pressure product at the moment of myocardial ischaemia during progressive exercise. Black circles, patients without exercise induced angina; white circles, patients with exercise induced angina; grey circles, mean of all patients' data. ${ }^{*} p<0.05$ pyridostigmine versus placebo. Data are mean (SE). drug's vagotonic action was lost during exercise and brief ischaemia. Also, no antiarrhythmic action was observed. The authors concluded that the actions of low dose pirenzepine and atenolol are similar during rest and that both drugs can be taken by patients who cannot tolerate $\beta$ blockade.

Although there is clinical evidence that a vagotonic action may be helpful for patients with heart disease, pyridostigmine seems to have several key advantages over other pharmacological options. Pyridostigmine does not cross the blood-brain barrier, it causes only minor and self limited side effects, it is administered orally, and, contrary to atropine, scopolamine, and pirenzepine, its parasympathetic action is dose dependent and thus can be individually adjusted.

Concerning the potential for practical application of pyridostigmine in the clinical setting, it was shown recently that patients with heart failure have more pronounced heart rate recovery after exercise when treated with pyridostigmine. ${ }^{30}$ This heart rate response is mediated by cholinergic activation and has been shown to identify patients at higher risk of cardiac events. ${ }^{31}$ In addition, pyridostigmine increased heart rate variability and diminished ventricular extrasystolic activity in patients with heart failure, suggesting that cholinergic activation with pyridostigmine may have a protective action. ${ }^{11}$ In the present study involving patients with coronary disease, pyridostigmine blunted the chronotropic response to exercise delaying the occurrence of myocardial ischaemia and leading to higher peak $\dot{\mathrm{V}}_{2}$. ST segment depression during exercise has been used for decades, not only for the diagnosis of coronary artery disease but also to indicate poor prognosis for patients with known disease. ${ }^{31-33}$ In addition, peak $\dot{\mathrm{V}}_{2}$ is the most important negative risk factor for cardiovascular and overall mortality, as documented by various studies of large number of patients. ${ }^{34-38}$ The key role of peak exercise capacity to predict long term mortality has been shown in patients with no heart disease $^{34}$ and in groups of patients with suspected ${ }^{35}{ }^{36}$ or with established coronary artery disease..$^{37}$ In the present study, the patients had ST segment depression at higher exercise intensity and achieved a higher peak $\dot{\mathrm{V}}_{2}$ with pyridostigmine, an effect that may provide relevant clinical benefits. Future prospective trials should investigate whether these effects of pyridostigmine correspond to actual protection in these patients.

\section{Conclusions}

Pyridostigmine improved peak exercise tolerance and inhibited the chronotropic response to submaximum exercise, increasing the intensity at which myocardial ischaemia occurred. These results suggest that pyridostigmine can protect patients with coronary disease against exercise induced myocardial ischaemia. 


\section{ACKNOWLEDGEMENTS}

Conselho Nacional de Desenvolvimento Científico e Tecnológico (CNPq), Brazil, and Fundação Carlos Chagas Filho de Amparo à Pesquisa do Estado do Rio de Janeiro (FAPERJ), Brazil.

\section{Authors' affiliations}

R R T Castro*, A C L Nóbrega*, Department of Physiology and Pharmacology, Universidade Federal Fluminense, Niterói, Brazil G Porphiriot, S M Serrat, Post-Graduate Program in Cardiology, Universidade Federal Fluminense, Niterói, Brazil

*Also Post-Graduate Program in Cardiology, Universidade Federal Fluminense, Brazil

†Also Aloysio de Castro State Institute of Cardiology, Rio de Janeiro, Brazil

\section{REFERENCES}

1 La Rovere MT, Bigger JT Jr, Marcus Fl, et al. Baroreflex sensitivity and heartrate variability in prediction of total cardiac mortality after myocardial infarction. Lancet 1998;351:478-84.

2 Schwartz PJ, La Rovere MT, Vanoli E. Autonomic nervous system and sudden death: experimental basis and clinical observations for post-myocardial infarction risk stratification. Circulation 1992;85:177-99.

3 Bigger JT, Coromilas J. How do beta-blockers protect after myocardial infarction? Ann Intern Med 1984;101:256-8.

4 Kleiger RE, Miller JP, Bigger JTJ, et al. Decreased heart rate variability and its association with increased mortality after acute myocardial infarction. Am J Cardiol 1987;59:256-62

5 Odemuyiwa O, Malik M, Farrel TG, et al. A comparison of the predictive characteristics of heart rate variability and left ventricular ejection fraction for all-cause mortality, arrhythmic events and sudden death after acute myocardial infarction. Am J Cardiol 1991;64:434-9.

6 Nóbrega ACL, Castro RRT. Parasympathetic dysfunction as a risk factor in myocardial infarction: what is the treatment? Am Heart J 2000; 140:e20.

7 Nóbrega AC, Carvalho AC, Bastos BG. Resting and reflex heart rate responses during cholinergic stimulation with pyridostigmine in humans. Braz J Med Biol Res 1996;29:1461-5.

8 Nóbrega AC, dos Reis AF, Moraes RS, et al. Enhancement of heart rate variability by cholinergic stimulation with pyridostigmine in healthy subjects. Clin Auton Res 2001;11:11-7.

9 Nóbrega ACL, Carvalho ACG, Santos KB, et al. Cholinergic stimulation with pyridostigmine blunts the cardiac responses to mental stress. Clin Auton Res 1999;9:1-6.

10 Serra SM, Vivacqua R, Ramalho SHR, et al. Exercise stress testing in healthy subjects during cholinergic stimulation after a single dose of pyridostigmine. Ara Bras Cardiol 2001;76:279-84.

11 Behling A, Moraes RS, Rohde LE, et al. Cholinergic stimulation with pyridostigmine reduces ventricular arrhythmia and enhances heart rate variability in heart failure. Am Heart J 2003;146:494-500.

12 Franklin BA. ACSM's guidelines for exercise testing and prescription. Philadelphia: Lippincott Williams \& Wilkins, 2000.

13 Vanhees L, Fagard R, Thiis L, et al. Prognostic significance of peak exercise capacity in patients with coronary artery disease. J Am Coll Cardiol 1994;23:358-63.

14 Myers JN. Information from ventilatory gas exchange data. In: Myers JN. Essentials of cardiopulmonary exercise testing. Champaign: Human Kinetics, 1996:83-108.

15 Castro RRT, Serra SM, Nóbrega ACL. Reduction of QTc interval dispersion: potential mechanism of cardioprotection of pyridostigmine bromide. Arq Bras Cardiol 2000;75:210-3.
16 Pontes PV, Nóbrega ACL, Mesquita ET, et al. Cholinergic stimulation with pyridostigmine, hemodynamic and echocardiographic analysis in healthy subjects. Arq Bras Cardiol 1999;72:302-6.

17 Grabe-Guimarães A, Alves LM, Tibiriçá $E$, et al. Pyridostigmine blunts the increases in myocardial oxygen demand elicited by the stimulation of the central nervous system in anesthetized rats. Clin Auton Res 1999;9:83-9.

18 Castro RRT, Porphyrio G, Serra SM, et al. Cholinergic stimulation with pyridostigmine reduces QTc interval in coronary artery disease. Braz J Med Biol Res 2002;35:685-9.

19 Ahnve S, Helmers C, Lundman T, et al. QTc intervals in acute myocardial infarction: first-year prognostic implications. Clin Cardiol 2000;3:303-8.

20 Arad M, Roth A, Zelinger J, et al. Safety of pyridostigmine in hypertensive patients receiving beta-blockers. Am J Cardiol 1992;15:518-22.

21 Stephenson A, Kolka MA. Acetylcholinesterase inhibitor, pyridostigmine bromide, reduces skin blood flow in humans. Am J Physiol 1990;258:951-7.

22 Marraccini $P$, Orsini E, Nassi G, et al. Effects of parasympathetic blockade on ischemic threshold in patients with exercise-induced myocardial ischemia. Am J Cardiol 1991;68:539-42.

23 Dibner-Dunlap ME, Eckberg DL, Magid MN, et al. The long-term increase of baseline and reflexly augmented levels of human vagal-cardiac activity induced by scopolamine. Circulation 1985;71:797-804.

24 La Rovere MT, Mortara A, Pantaleo P, et al. Scopolamine improves autonomic balance in advanced congestive heart failure. Circulation 1994;90:838-43.

25 Jakubetz J, Schmuck S, Wochatz G, et al. Human cardiac $\beta_{1^{-}}$or $\beta_{2^{-}}$ adrenergic receptor stimulation and the negative chronotropic effect low-dose pirenzepine. Clin Pharmacol Ther 2000;67:549-57.

26 Hayano T, Shimizu A, Ikeda Y, et al. Paradoxical effects of pirenzepine on parasympathetic activity in chronic heart failure and control. Int J Cardiol 1999;68:47-56.

27 Meyer EC, Sommers DK. Possible mechanisms of anti-cholinergic druginduced bradycardia. Eur J Clin Pharmacol 1998;35:503-6.

28 Marraccini P, Orsini E, Nassi G, et al. Usefulness of pirenzepine, an M1 antimuscarinic agent, for effort myocardial ischemia. Am J Cardiol 1992:69:1407-11.

29 Pedretti RFE, Prete G, Foreman RD, et al. Autonomic modulation during acute myocardial ischemia by low-dose pirenzepine in conscious dog with a healed myocardial infarction: a comparison with $\beta$-adrenergic blockade. J Cardiovasc Pharmacol 2003:41:671-7.

30 Androne AS, Hryniewicz K, Goldsmith R, et al. Acetylcholinesterase inhibition with pyridostigmine improves heart rate recovery after maximal exercise in patients with chronic heart failure. Heart 2003;89:854-8.

31 Cole CR, Blackstone EH, Pashkow FJ, et al. Heart-rate recovery immediately after exercise as a predictor of mortality. N Engl J Med 1999;341:1351-7.

32 McNeer JF, Margolis JR, Lee KL, et al. The role of the exercise test in the evaluation of patients for ischemic heart disease. Circulation 1978;57:64-70.

33 Weiner DA, McCabe CH, Ryan TJ. Identification of patients with left main and three vessel coronary disease with clinical and exercise test variables. Am J Cardiol 1980;46:21-7.

34 Laukkanen JA, Lakka TA, Rauramaa R, et al. Cardiovascular fitness as a predictor of mortality in men. Arch Intern Med 2001;161:825-31.

35 Prakash M, Myers J, Froelicher VF, et al. Clinical and exercise test predictors of all-cause mortality: results from $>6,000$ consecutive referred male patients. Chest 2001;120:1003-13

36 Herlitz J, Karlson BW, Lindqvist J, et al. Prognosis and risk indicators of death during a period of 10 years for women admitted to the emergency department with a suspected acute coronary syndrome. Int J Cardiol 2002;82:259-68.

37 Kavanagh T, Mertens DJ, Hamm LF, et al. Prediction of long-term prognosis in 12169 men referred for cardiac rehabilitation. Circulation 2002;106:666-71.

38 Ghayoumi A, Raxwal V, Cho S, et al. Prognostic value of exercise tests in male veterans with chronic coronary artery disease. J Cardiopulm Rehabil 2002;22:399-407. 